\title{
Evaluation of collective transport properties of ionic melts from molecular dynamics simulations ${ }^{\dagger}$
}

\author{
MANISH AGARWAL and CHARUSITA CHAKRAVARTY* \\ Department of Chemistry, Indian Institute of Technology Delhi, New Delhi 110016 \\ e-mail: charus@chemistry.iitd.ernet.in
}

\begin{abstract}
Molecular dynamics simulations of beryllium fluoride $\left(\mathrm{BeF}_{2}\right)$ have been carried out in the canonical (NVT) ensemble using a rigid-ion potential model. The Green-Kubo formalism has been applied to compute viscosities and ionic conductivities of $\mathrm{BeF}_{2}$ melt. The computational parameters critical for reliably estimating these collective transport properties are shown to differ significantly for viscosity and ionic conductivity. In addition to the equilibrium values of these transport properties, structural relaxation times as well as high-frequency IR-active modes are computed from the pressure and charge-flux auto correlation functions (ACFs) respectively. It is shown that a network-forming ionic melt, such as $\mathrm{BeF}_{2}$, will display persistent oscillatory behaviour of the integral of the charge-flux ACF. By suitable Fourier transformation, one can show that these persistent oscillations correspond to highfrequency, infra-red active vibrations associated with local modes of the network.
\end{abstract}

Keywords. Ionic melts; molecular dynamics; viscosity; ionic conductivity.

\section{Introduction}

Ionic melts are an interesting class of materials which are of considerable technological significance. ${ }^{1-3}$ From the point of view of liquid state structure and dynamics, an ionic melt can be considered as a mixture of atoms with very different electronegativities. The relative importance of electrostatic and covalent interactions will depend on the relative electronegativities and polarizabilities. Molten alkali halides (other than those of lithium) will be dominated by Coulombic interactions with short-range repulsion and dispersion interactions playing an additional role in determining local structure. The introduction of local, anisotropic covalent interactions results in the formation of liquid state networks of varying extent, dimensionality and local order. Examples of such network-forming ionic melts include $\mathrm{ZnCl}_{2}$, $\mathrm{BeF}_{2}, \mathrm{SiO}_{2}, \mathrm{GeO}_{2}, \mathrm{AlCl}_{3}$ and $\mathrm{GeSe}_{2}{ }^{4-19}$ Understanding the transport properties of ionic melts, and their relationship with the underlying liquid state structure, is of considerable current interest from the point of view of both experiment and theory. Transport properties can be computed from the equilibrium or non-equilibrium molecular dynamics simulations. ${ }^{20-23}$ Diffusivity is the transport property of ionic melts

${ }^{\dagger}$ Dedicated to the memory of the late Professor S K Rangarajan *For correspondence which is most frequently and conveniently estimated from molecular dynamics simulations. Since the diffusivity is a single-particle quantity, reliable estimates can be obtained from equilibrium molecular dynamics simulations using either the Einstein relation for the mean square displacement or the equivalent Green-Kubo formulation involving the integral of the single-particle velocity autocorrelation function. The collective transport properties of ionic melts which are of experimental interest are the viscosity, the thermal conductivity and the ionic conductivity. If collective effects are insignificant, then these quantities can be estimated from the diffusivity. For example, the Stokes-Einstein relation connects the viscosity with self diffusivities:

$$
D_{ \pm}=k_{B} T / 4 \pi \eta r_{ \pm}
$$

where $r_{+}$and $r_{-}$are the cationic and anionic radii respectively, and the factor of 4 originates from the use of 'slip' boundary conditions. Similarly, the NernstEinstein relation relates the ionic conductivity:

$$
\sigma=\left(e^{2} \rho / k_{B} T\right)\left(x_{+} z_{+}^{2} D_{+}+x_{-} z_{-}^{2} D_{-}\right)(1-\Delta),
$$

where $\mathrm{e}$ is the electronic charge, $x_{ \pm}$and $z_{ \pm}$are the ionic mole fractions and charges, respectively and $\Delta$ is the Nernst-Einstein deviation factor. The StokesEinstein and Nernst-Einstein relations are approximately valid for many ionic melts; for example, the 
mean value of the Nernst-Einstein parameter for alkali halides is 0.26 which is small but not insignificant. ${ }^{24}$ It is reasonable to expect that the deviations will be much larger for network-forming ionic melts reflecting the sensitivity of collective transport properties, such as viscosity and the ionic conductivity, to multi-particle structural and dynamical correlations. These collective transport properties, however, are more prone to statistical error when estimated from molecular dynamics simulations and typically require longer and more careful simulations. As a consequence, they have been directly evaluated from MD simulations for a very limited range of ionic melts. ${ }^{18,25,26}$

In the present paper, we address the computation of shear viscosity, $\eta$ and ionic conductivity, $\sigma$ for network-forming ionic melts. As an illustrative example of a network-forming melt with significant multiparticle structural and dynamical correlations, we choose beryllium fluoride $\left(\mathrm{BeF}_{2}\right)$. Liquid $\mathrm{BeF}_{2}$, like the well-studied $\mathrm{SiO}_{2}$ melt, is a tetrahedral, network-forming ionic melt and shows liquid-state anomalies similar to those of water. ${ }^{27-29}$ Such anomalous properties include rise in density on isobaric heating (density anomaly) and a rise in diffusivity on isothermal compression (diffusional anomaly). In the case of $\mathrm{BeF}_{2}$, we have recently carried out detailed studies of the thermodynamic anomalies and the associated behaviour of structural order metrics. ${ }^{30-32}$ With regard to mobility anomalies, other than an initial study of diffusivity along a single isochore, there are no simulation studies of transport properties. ${ }^{18}$ In this paper, we address the efficient computation of collective transport properties for this system, focusing specifically on viscosity and ionic conductivity. Computation of transport properties can be done using either equilibrium or non-equilibrium molecular dynamics simulations. Since the equilibrium MD approach can be readily combined with simulations to determine the phase diagram and other thermodynamic properties, we use an equilibrium MD approach based on Green-Kubo formalism for estimating transport properties from integrals of the appropriate autocorrelation functions (ACFs). We use a rigid-ion TRIM potential energy surface for modelling the interactions in $\mathrm{BeF}_{2}$ melt. ${ }^{18,19,33,34}$ The TRIM model neglects ionic polarizabilities and there has been considerable effort recently in developing polarizable potentials based on $a b$ initio molecular dynamics simulations. The comparison of TRIM and polarizable models suggests that the TRIM model is semi- quantitatively reasonable though it does tend to overestimate pressure ${ }^{30,35-37}$ It is interesting to note that despite the absence of ionic polarizability to model covalent bond formation, or three-body potentials to mimic bond angles constraints, TRIM and related potential models are able to generate correct type of local order (tetrahedral for $\mathrm{BeF}_{2}$ ) due to a combination of formal charge assignments and effective ionic radius ratios. ${ }^{15}$ The paper is organised as follows. Section 2 contains computational details, including the Green-Kubo relations to estimate viscosity and ionic conductivity. The results and conclusions are presented in section 3 and 4 respectively.

\section{Computational details}

\section{$2.1 \quad$ Potential model}

We use the transferable rigid ion model (TRIM) potential to model interatomic interactions in beryllium fluoride since it has been extensively used to study a range of ionic melts. ${ }^{33}$ The TRIM potential is pair-additive and models electrostatic long-range interactions with Coulomb interactions and shortrange repulsions using a Born-Mayer-Huggins parametric form. The pair interaction between ions $i$ and $j$ is given by:

$$
\begin{aligned}
\phi_{\text {TRIM }}\left(r_{i j}\right)= & \frac{z_{i} z_{j} e^{2}}{4 \pi \varepsilon_{o} r_{i j}}+\left(1+\frac{z_{i}}{n_{i}}+\frac{z_{j}}{n_{j}}\right) \\
& \times b \exp \left(\frac{\sigma_{i}+\sigma_{j}-r_{i j}}{s}\right),
\end{aligned}
$$

where $r_{i j}$ is the distance between atoms $i$ and $j$. The parameters associated with an atom of type $l$ are the charge $z_{l}$, the number of valence-shell electrons $n_{l}$ and the ionic size parameter $\sigma_{l}$. The repulsion parameter $b$ and the softness parameter $s$ are assumed to be the same for all three types of pair interactions. In the case of $\mathrm{BeF}_{2}$, there are two types of ions and three types of pair interactions that must be considered for the TRIM parameterization of $\mathrm{BeF}_{2}$ i.e. $\mathrm{Be}^{2+}-\mathrm{Be}^{2+}, \mathrm{Be}^{2+}-\mathrm{F}^{-}, \mathrm{F}^{-}-\mathrm{F}^{-}$. The corresponding parameters for $\mathrm{BeF}_{2}$ used in this work are given in table 1 and have been used in previous studies. ${ }^{18,19,34}$

\subsection{Molecular dynamics}

Molecular dynamics simulations of a system of 150 $\mathrm{Be}$ and $300 \mathrm{~F}$ ions were carried out in canonical 
Table 1. TRIM potential parameters for $\mathrm{BeF}_{2}{ }^{18,19,34}$

\begin{tabular}{cccccccc}
\hline$\sigma^{+}(\AA)$ & $\sigma^{-}(\AA)$ & $s(\AA)$ & $z^{+}$ & $z^{-}$ & $N^{+}$ & $n^{-}$ & $b\left(\mathrm{~kJ} \mathrm{~mol}^{-1}\right)$ \\
\hline 0.93 & 1.24 & 0.29 & 2 & -1 & 2 & 8 & 34.33 \\
\hline
\end{tabular}

(NVT) ensemble under cubic periodic boundary conditions, using the DL_POLY software package. ${ }^{38,39}$ The long-range effects of electrostatic interactions were accounted for by the Ewald summation method. ${ }^{21,23}$ A Berendsen thermostat, with the time constant $\tau_{\mathrm{B}}=200 \mathrm{ps}$, was used to maintain the desired temperature for the production run. The thermostat time constant was chosen to be large so that the perturbation of the intrinsic system dynamics is small and transport properties can be evaluated. The Verlet leapfrog algorithm with a time step of $1 \mathrm{fs}$ was used to integrate the equations of motion. Starting liquid $\mathrm{BeF}_{2}$ configurations were generated by replacing corresponding ions in an equilibrated low temperature $\mathrm{SiO}_{2}$ configuration. ${ }^{29}$ The system thus obtained was heated gradually to form the equilibrated liquid state and an MD simulation at each state point was performed for $0.1 \mathrm{~ns}$ with target temperatures changing from $1000 \mathrm{~K}$ to $6000 \mathrm{~K}$ in steps of $100 \mathrm{~K}$ each. The results for static equilibrium averages for quantities such as the configurational energy and pressure from these $0.1 \mathrm{~ns}$ is in agreement with previous results within the statistical error bars. ${ }^{18}$ Longer equilibration and production runs were carried out for a subset of about 100 state points covering the entire range in temperature and density. ${ }^{30,31}$ For temperatures above $2500 \mathrm{~K}$, equilibration runs of $6 \mathrm{~ns}$, followed by production runs of 6 ns with data dumping every 5 time steps were performed. For temperatures at and below $2250 \mathrm{~K}$, equilibration runs were $10 \mathrm{~ns}$, followed by production runs of $8 \mathrm{~ns}$ for viscosity estimates, with data stored at every 2 steps. For ionic conductivity estimation, short runs of $0.5 \mathrm{~ns}$ were done, with data stored at every time step. The system was simulated at 7 temperatures in the $1500 \mathrm{~K}$ to $3000 \mathrm{~K}$ range along 14 to 16 isochores in the density range from $1.5 \mathrm{~g} \mathrm{~cm}^{-3}$ to $3.0 \mathrm{~g} \mathrm{~cm}^{-3}$ with gaps of $250 \mathrm{~K}$ and $0 \cdot 1 \mathrm{~g} \mathrm{~cm}^{-3}$ respectively.

\subsection{Shear viscosity}

Conventional pressure, $p$, is the force per unit area, applied in a direction perpendicular to the surface. However, in a viscous liquid such as $\mathrm{BeF}_{2}$, accurate description of the pressure involves contributions from components along the cartesian directions. ${ }^{20}$ Hence, the 'true' pressure is a rank 2 tensor with elements $P_{\alpha \beta}$, which denotes the force acting along direction $\alpha$ on a unit surface perpendicular to the direction $\alpha$, where $\alpha$ and $\beta$ are the cartesian directions $x, y$ or $z$. The scalar pressure $p$ is the trace of the pressure tensor $P$.

The instantaneous value of pressure tensor component $P_{\alpha \beta}$ at time $t$ is given by

$$
P_{\alpha \beta}(t)=\left\langle\frac{1}{V} \sum_{i=1}^{n_{a t}} m_{i} v_{i \alpha} v_{i \beta}+\sum_{i=1}^{n_{a t}} \sum_{j>i}^{n_{a t}} f_{\alpha i j}\left(r_{i \beta}-r_{j \beta}\right)\right\rangle,
$$

where $v_{i \alpha}$ and $r_{i \alpha}$ are the velocity and position along direction $\alpha$ of the $i$ th atom having mass $m_{i}$ and $f_{\alpha i j}$ is the force between atoms $i$ and $j$ along direction $\alpha$. Since the potential energy surface is pair additive, the pressure tensor can be assumed to be symmetric i.e. $P_{x y}=P_{y x}{ }^{20}$ The shear viscosity, $\eta$, is then given by the Green-Kubo relation corresponding to the time integral of the off-diagonal pressure autocorrelation function (ACF):

$$
\eta=\frac{V}{k_{B} T} \int_{0}^{\infty}\left\langle P_{\alpha \beta}(0) P_{\alpha \beta}(t)\right\rangle \mathrm{d} t,
$$

where the ensemble average uses the mean of the three unique off-diagonal pressure tensor elements, namely $P_{x y}, P_{y z}$ and $P_{z x}$. Unlike in the case of the corresponding Einstein relation the periodic boundary conditions do not have to be explicitly accounted for when evaluating the above expression. ${ }^{20,21}$ The time constant of the Berendsen thermostat must be chosen to be greater than the correlation time $\left(t_{w}\right)$ of the autocorrelation function.

The running integral of the off-diagonal pressure autocorrelation function (ACF), defined in (5), is plotted as a function of time for several state points in figure 1. An approximate estimate of the correlation time can be obtained by noting the time taken for the integral to reach a plateau value. A stable plateau value is typically obtained within $1-2$ ps at high temperatures and $4-5 \mathrm{ps}$ for low temperatures. 
Small oscillations about the mean plateau value remain even at long times. In order to estimate the viscosity and the associated error, we have followed the standard procedure in the literature and calculated the mean value associated with the terminal value of the integral of the plateau region. The error was estimated by dividing the 6-8 ns trajectory into non-overlapping $1 \mathrm{~ns}$ sub- trajectories and obtaining separate estimates $\eta$ from each of the sub-trajectories by the above procedure. The critical computational parameters for estimating the viscosity are: (a) $t_{d}$ is the total length of simulation performed; (b) $t_{w}$ is the length of the time window over which the integral of the ACF is performed and (c) $t_{s}$ is the time difference between successive windows each of length $t_{w}$. For a given sub-trajectory, the ACF is obtained by averaging over all associated windows. Table 2 shows the values of these parameters used for viscosity estimations of $\mathrm{BeF}_{2}$ in this work and in the

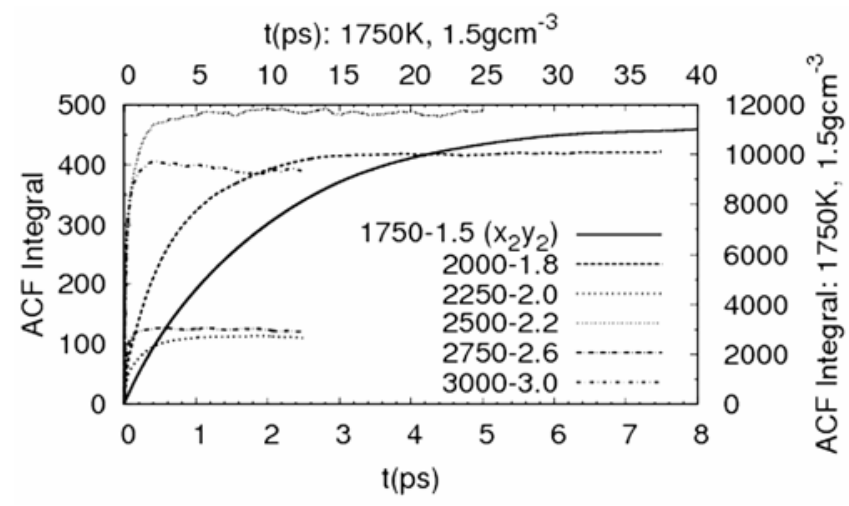

Figure 1. Running integral of the pressure autocorrelation function, for different state points $(T, \rho)$. $T$ is given in Kelvin, and $\rho$ in $\mathrm{g} \mathrm{cm}^{-3}$. Note that the time for reaching a plateau for $1750 \mathrm{~K}, 1.5 \mathrm{~g} \mathrm{~cm}^{-3}$ is a magnitude higher than those at higher densities and temperatures, and hence the corresponding time and $\mathrm{ACF}$ scales are given on the top and right axes respectively of the graph.

Table 2. Comparison of Green-Kubo integration parameters: $t_{\mathrm{d}}$ is the total length of simulation performed; $t_{\mathrm{w}}$ is the length of the time window over which the integrals from (5) and (6) are evaluated; $t_{\mathrm{s}}$ is the difference between the origins of successive time windows.

\begin{tabular}{llll}
\hline & \multicolumn{1}{c}{$t_{\mathrm{d}}$} & \multicolumn{1}{c}{$t_{\mathrm{w}}$} & \multicolumn{1}{c}{$t_{\mathrm{s}}=\Delta t_{\text {dump }}$} \\
\hline $\mathrm{NaCl}(\eta)^{26}$ & $0 \cdot 1-10 \mathrm{~ns}$ & $2 \mathrm{ps}$ & $1 \mathrm{fs}-100 \mathrm{ps}$ \\
$\mathrm{BeF}_{2}(\eta)$ & $6-8 \mathrm{~ns}$ & $2 \cdot 5-40 \mathrm{ps}$ & $2-10 \mathrm{fs}$ \\
$\mathrm{NaCl}^{4}(\sigma)^{41}$ & $72-300 \mathrm{ps}$ & - & - \\
$\mathrm{BeF}_{2}(\sigma)$ & $500 \mathrm{ps}$ & $1 \mathrm{ps}-2 \mathrm{ps}$ & $1 \mathrm{fs}$ \\
\hline
\end{tabular}

study by Nevins and Spera on viscosity of $\mathrm{NaCl}^{26}{ }^{26} \mathrm{It}$ may be noted that $t_{w}$ is significantly less than $t_{d}$; this is because computing the running integral for longer times did not show any significant decrease in error. We also use the pressure ACFs to obtain an estimate of the structural relaxation times for the liquid. Two different estimators are used: (a) relaxation time, $\tau$, obtained by least square fitting of the ACF to the function $\exp \left(\left(t^{\prime}-t\right) / \tau\right)$ and (b) the Maxwell time $\tau_{\text {Maxwell, }}$, such that $\eta\left(\eta_{\text {Maxwell }}\right) / \eta(\eta \rightarrow \infty)=1-(1 / e)$.

\subsection{Ionic conductivity}

The Green-Kubo relation for the ionic conductivity, $\sigma$, is defined as: ${ }^{40}$

$$
\sigma=\frac{1}{V k_{B} T} \int_{0}^{\infty}\langle j(t) \cdot j(0)\rangle \mathrm{d} t
$$

where $V$ and $T$ are the volume and temperature of the system respectively and the charge flux $j(t)$ is defined by

$$
j(t)=\sum_{i=1}^{n} z_{i} e v_{i}(t)
$$

where, $z_{i} e$ and $v_{i}$ are the charge and velocity of atom $i$ respectively. The charge flux was stored for $5 \times 10^{5}$ consecutive steps. Except at very low densities, the correlation times for the charge flux autocorrelation function were very similar to those for the viscosity i.e. at $4-5 \mathrm{ps}$ for low temperatures and $1-2 \mathrm{ps}$ for high temperatures. The oscillations about the plateau value of the integral were much less than $1 \%$ for most state points. At low temperatures and densities, however, the integral of the charge ACF shows persistent, nearly sinusoidal oscillations with amplitude of about $6-10 \%$ of the mean plateau value. The critical parameters for the estimation of the conductivity are analogous to those for the viscosity and are given in table 2 for $\mathrm{BeF}_{2}$ and $\mathrm{NaCl}$ melts. The electric charge-flux correlation function also contains the information about the dipole moment correlation function. ${ }^{14}$ One can therefore take the Fourier transform of the electric charge flux correlation function to obtain the infrared spectrum of the melt:

$$
I(\omega)=\int_{0}^{\infty} d t e^{i \omega t}\langle j(t) \cdot j(0)\rangle
$$




\section{Results and discussion}

We have computed conductivities, viscosities and structural relaxation times for a set of state points shown in table 3 . The state points were chosen to represent both normal and anomalous thermodynamic regimes, with the high temperature-high density state points belonging to the normal regime and the low-density-low temperature points belonging to the anomalous regime. A more detailed description of the thermodynamic behaviour over the entire set of state points is given elsewhere. ${ }^{30}$ The two different estimators for the structural relaxation times, $\tau$ and $\tau_{\mathrm{M}}$, show the same trends but have somewhat different quantitative values. The structural relaxation times change by about an order of magnitude over the temperature range from $1750 \mathrm{~K}$ to $3000 \mathrm{~K}$, indicating that window lengths over which the auto-
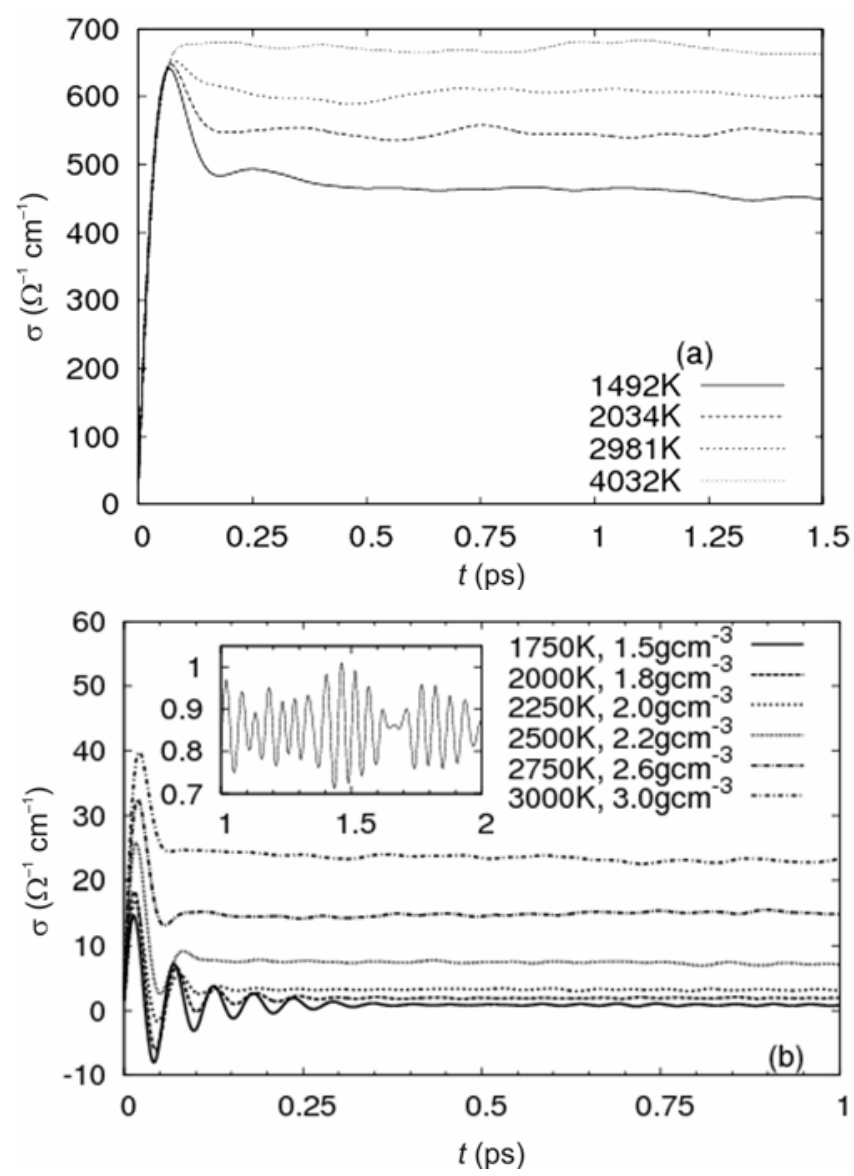

Figure 2. Running integral of the charge-flux autocorrelation function for different state points in the $\rho-$ $T$-plane for: (a) $\mathrm{NaCl}$ melt and (b) $\mathrm{BeF}_{2}$ melt. The oscillatory amplitude is small in comparison to the actual average value and inset shows the running integral for $\left(1750 \mathrm{~K}, 1.8 \mathrm{~g} \mathrm{~cm}^{-3}\right)$. correlation function must be integrated to obtain reliable simulation averages must be chosen accordingly. Figure 1 displays the running integral for estimation of shear viscosity, $\eta$ for all the state points. It is evident that the correlation length for the low temperature regime $(1500 \mathrm{~K}$ and $1750 \mathrm{~K}$ ) required is nearly 5 times of that required for higher temperatures, above $2000 \mathrm{~K}$. At low temperatures, smooth convergence of the pressure ACF integral is obtained and there is very little statistical noise or fluctuations in the value of the integral as a function of time. In contrast, at high temperatures, when the viscosity is small and the value of the integral decreases by a couple of orders of magnitude (see figure 1), the statistical noise is much more pronounced. The optimal size of the time window for which the integral of the pressure ACF is computed must be such that a stable plateau value is achieved but the effect of statistical noise in the plateau value at long times does not significantly increase the error.

The ionic conductivities for selected state points of $\mathrm{BeF}_{2}$ melt are shown in table 3. The behaviour of the integral for the charge-flux autocorrelation function to evaluate the ionic conductivity is shown in figure 2 for those state point of $\mathrm{BeF}_{2}$ melt, as well as for four state points of $\mathrm{NaCl}$ melt that are very close to those studied by Guissani and Guillot. ${ }^{41}$ For these state points, our results for $\sigma$ agree with their values, within statistical error. The correlation time for the charge-flux ACF is much shorter than for the pressure ACF, as reflected in our choice of $t_{\mathrm{w}}$. In the case of $\mathrm{NaCl}$ melt, the conductivity estimated as a function of the time for which the ACF is integrated, rapidly settles down to a stable plateau value with minimal fluctuations. $\mathrm{BeF}_{2}$ melt shows a similar behaviour in the normal regime at high temperatures i.e. the integral shows a sharp rise, a short decrease, and then stabilizes to a plateau with very little noise. At low temperatures and densities, specially prominent at $1750 \mathrm{~K}$ and $1.5 \mathrm{~g} \mathrm{~cm}^{-3}$, the integral shows pronounced oscillatory behaviour about the mean plateau value. The oscillations slowly decay from an initial high amplitude to a constant near the average value.

To understand the origin of the oscillatory behaviour of the charge-flux $\mathrm{ACF}$ in $\mathrm{BeF}_{2}$ melt, we compute the Fourier transform of the charge-flux ACF, as defined in (8). Figure 3 shows this Fourier transform for several densities along the $1750 \mathrm{~K}$ isotherm. This corresponds essentially to the infrared spectrum of the melt. Figure 3 shows the IR 
Table 3. Shear viscosity $(\eta)$ and ionic conductivity $(\sigma)$ for few representative state points. The structural relaxation time $\tau$ and the Maxwell time, $\tau_{\mathrm{M}}$, are also tabulated.

\begin{tabular}{|c|c|c|c|c|c|}
\hline $\mathrm{T}(\mathrm{K})$ & $\rho\left(\mathrm{g} \mathrm{cm}^{-3}\right)$ & $\eta(\mathrm{cP})$ & $\tau(\mathrm{ps})$ & $\tau_{\mathrm{M}}(\mathrm{ps})$ & $\sigma\left(\Omega^{-1} \mathrm{~cm}^{-1}\right)$ \\
\hline 1750 & $1 \cdot 8$ & $1.684 \pm 0.32$ & $2 \cdot 018$ & $2 \cdot 18$ & $1.022 \pm 0.077$ \\
\hline 2000 & $2 \cdot 0$ & $0.317 \pm 0.040$ & 0.376 & 0.29 & $2.799 \pm 0.12$ \\
\hline 2250 & $2 \cdot 2$ & $0.189 \pm 0.013$ & $0 \cdot 177$ & 0.096 & $6 \cdot 800 \pm 0 \cdot 12$ \\
\hline 2500 & $2 \cdot 4$ & $0.159 \pm 0.016$ & $0 \cdot 112$ & 0.045 & $11.06 \pm 0.18$ \\
\hline 2750 & $2 \cdot 6$ & $0 \cdot 147 \pm 0 \cdot 014$ & $0 \cdot 074$ & $0 \cdot 028$ & $15 \cdot 34 \pm 0 \cdot 94$ \\
\hline 3000 & $3 \cdot 0$ & $0.180 \pm 0.004$ & $0 \cdot 020$ & 0.025 & $24.77 \pm 1.04$ \\
\hline
\end{tabular}

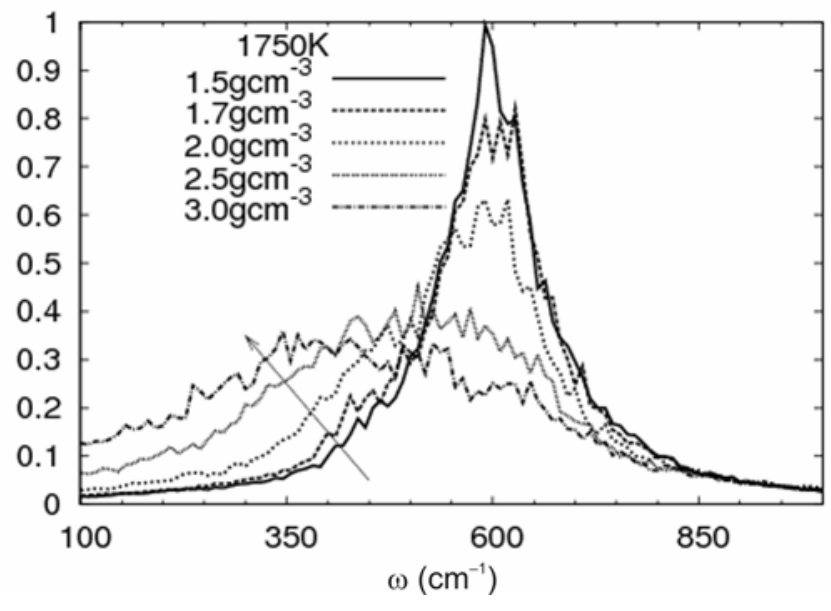

Figure 3. Fourier transform of the electric charge-flux auto correlation function, corresponding to the infrared spectrum for $\mathrm{BeF}_{2}$ melt, at different densities along the $1750 \mathrm{~K}$ isotherm. Arrow indicates direction of increasing density.

spectra for $\mathrm{BeF}_{2}$ at different densities along the $1750 \mathrm{~K}$ isotherm. The clear peak at $600 \mathrm{~cm}^{-1}$ for low densities $\left(<2.0 \mathrm{~g} \mathrm{~cm}^{-3}\right)$ decays to a broad maxima centered at $360 \mathrm{~cm}^{-1}$. This behaviour has been noted in earlier work where the focus was on computation of IR active modes in fluoroberyllate glasses. ${ }^{28,42} \mathrm{At}$ low densities, the local tetrahedral order is much more pronounced and the local vibrational modes are associated with relatively high frequencies. At higher densities, the tetrahedral packing is progressively destroyed as coordination numbers increase and this is reflected in the local vibrational frequencies. In our context, where the focus is on computation of conductivity, the existence of local vibrational modes is reflected in the high-frequency oscillations of the ACF integral at long times. This will clearly be characteristic of network-forming ionic melts. Ionic melts where network-formation is insignificant e.g. $\mathrm{BeF}_{2}$ in the normal regime and $\mathrm{NaCl}$ melt, do not show this type of behaviour. In the case of network-forming melts, we take a local average of the
ACF integral over approximately 500 successive time steps to minimise the effect of these oscillatory fluctuations and obtain a stable estimate for the conductivity.

\section{Conclusions}

In this paper, we apply the Green-Kubo formalism to compute viscosities and ionic conductivities of $\mathrm{BeF}_{2}$ melt, modelled using a rigid-ion pair potential. We show that when estimating these collective transport properties, considerable care has to be taken when in deciding the simulation parameters, such as the length of MD simulations, the frequency of sampling autocorrelation function data, the window length for which the autocorrelation function is integrated, and the method used for averaging. These simulation parameters differ significantly for viscosity and conductivity. In addition to the equilibrium values of these transport properties, we compute structural relaxation times as well as high-frequency IR-active modes from the pressure and charge-flux ACFs respectively. The charge-flux autocorrelation function typically shows a shorter correlation time than the pressure autocorrelation function. We also show that a network-forming ionic melt, such as $\mathrm{BeF}_{2}$, will display persistent oscillatory behaviour of the integral of the charge-flux ACF. To obtain stable values for the conductivity, one should take shorttime averages of the integral of the charge-flux ACF. By suitable Fourier transformation, one can show that these persistent oscillations correspond to high-frequency, infra-red active vibrations associated with local modes of the network. In the absence of a liquid-state network, as in $\mathrm{NaCl}$ melt or in $\mathrm{BeF}_{2}$ at high temperatures, this behaviour is absent. Our results demonstrate that the Green-Kubo route provides an efficient method for estimating transport properties of ionic melts from equilibrium molecular dynamics simulations. 


\section{References}

1. Madden P A and Wilson M 2000 J. Phys. Cond. Matt. 12 A95

2. McMillan P F 2004 J. Mater. Chem. 141506

3. McMillan P F, Wilson M, Wilding M C, Daisenberger D, Mezouar M and Greaves G N 2007 J. Phys. Cond. Matt. 19415101

4. Wilson M and Madden P A 1998 Phys. Rev. Lett. 80 532

5. Micoulaut M, Cormier L and Henderson G S 2006 J. Phys. Cond. Matt. 18 R753

6. Shanavas K V, Garg N and Sharma S M 2006 Phys. Rev. B73 094120

7. Brawer S A 1980 Phys. Rev. Lett. 46778

8. van der Meer J R M and Konings R J M $2007 \mathrm{~J}$. Nucl. Mater. 36016

9. Scala A, Starr F W, La Nave E, Sciortino F and Stanley H E 2000 Nature 406166

10. Ford M H, Auerbach S M and Monson P A 2004 J. Chem. Phys. 1218415

11. Wright A C, Clare A G, Etherington G, Sinclair R N, Brawer S A and Weber M J 1989 J. Non-Cryst. Solids 111139

12. Hawlitzky M, Horbach J, Ispas $S$, Krack $M$ and Binder K 2008 J. Phys. Cond. Matt. 20285106

13. Poole P H, Hemmati M and Angell C A 1997 Phys. Rev. Lett. 792281

14. Wilson M, Madden P A, Hemmati M and Angell C A 1996 Phys. Rev. Lett. 774023

15. Takada A, Richet P, Catlow C R A and Price G D 2007 J. Non-Cryst. Solids 3531892

16. Saika-Voivod I, Sciortino F and Poole P H 2000 Phys. Rev. E63 011202

17. Shell M S, Debenedetti P G and Panagiotopoulos A Z 2002 Phys. Rev. E66 011202

18. Hemmati M, Moyinihan C T and Angell C A 2001 J. Chem. Phys. 1156663

19. Hemmati M and Angell C A 1997 J. Non-Cryst. Solids 217236

20. Rapaport D C 1998 The art of molecular dynamics simulation (Cambridge: Cambridge University Press)
21. Frenkel D and Smit B 2002 Understanding molecular simulation: From algorithms to applications (London: Academic Press)

22. McQuarrie D A 2000 Statistical mechanics (University Science Books)

23. Allen M D and Tildesley D F 1986 Computer simulation of liquids (Oxford: Clarendon Press)

24. Hansen J-P and McDonald I R 1986 Theory of simple liquids (Academic Press)

25. Galamba N, de Castro C A N and Ely J F 2004 J. Chem. Phys. 1208676

26. Nevins D and Spera F J 2007 Mol. Simulat. 331261

27. Tanaka H 2002 Phys. Rev. B66 064202

28. Angell C A, Bressel R D, Hemmati M, Sare E J and Tucker J C 2000 Phys. Chem. Chem. Phys. 21559

29. Sharma R, Chakraborty S N and Chakravarty C 2006 J. Chem. Phys. 125204501

30. Agarwal M, Sharma S and Chakravarty C 2007 J. Chem. Phys. 127164502

31. Agarwal M and Chakravarty C 2007 J. Phys. Chem. B111 13294

32. Sharma R, Agarwal M and Chakravarty C $2008 \mathrm{Mol}$. Phys. 1061935

33. Tosi M P and Fumi F G 1964 J. Phys. Chem. Solids 25 31; Tosi M P and Fumi F G $1964 J$. Phys. Chem. Solids 2545

34. Woodcock L V, Angell C A and Cheeseman P 1976 J. Chem. Phys. 651565

35. Salanne M, Simon C, Turq P, Heaton R J and Madden P A 2006 J. Phys. Chem. B110 11461

36. Salanne M, Simon M, Turq P and Madden P A 2007 J. Phys. Chem. B111 4678

37. Heaton R J, Brookes R, Madden P A, Salanne M, Simon C and Turq P 2006 J. Phys. Chem. B110 11454

38. Smith W and Forester T R 1996 J. Mol. Graphics 14136

39. Smith W, Yong C W and Rodger P M $2002 \mathrm{Mol}$. Simulat. 28 385; The DL_POLY website is http:// www.cse.clrc.ac.uk/msi/software/DL POLY/

40. Gillan M J 1991 Physica Scripta t39 $\overline{3} 62$

41. Guissani Y and Guillot B 1994 J. Chem. Phys. 101 490

42. Boulard B, Kieffer K, Phifer C C and Angell C A 1992 J. Non-Cryst. Solids 140250 